\title{
Prevalencia de Parasitosis Intestinales y Factores Asociados en un Corregimiento de la Costa Atlántica Colombiana
}

\section{Prevalence of intestinal parasitism and associated factors in a village on the Colombian Atlantic Coast}

\author{
Sonia Agudelo-Lopez ${ }^{1}$, Lucila Gómez-Rodríguez ${ }^{2}$, Xiomara Coronado², Adalina \\ Orozco $^{2}$, Carlos A. Valencia-Gutierrez ${ }^{2}$, Luis F. Restrepo-Betancur ${ }^{1}$, \\ Luisa A. Galvis-Gómez y Luz E. Botero-Palacio ${ }^{1}$
}

1 Facultad de Medicina Universidad de Antioquia. pilaragudelo@yahoo.com, lusitano@agronica.udea.edu.co; lagalvisg@gmail.com

2 Universidad Metropolitana de Barranquilla. lucycgomez@hotmail.com; xcoronado@latinmail.com; carlosvalencia@yahoo.com

Recibido 21 Noviembre 2007/Enviado para Modificación 28 Junio 2008/Aceptado 2 Septiembre 2008

\section{RESUMEN}

Objetivos Determinar la prevalencia del parasitismo intestinal e identificar los factores de riesgo asociados a estos, en el corregimiento de Loma Arena, Departamento de Bolívar, Colombia

Metodología Mediante encuesta aplicada a cada grupo familiar, fueron evaluadas las condiciones socio-sanitarias y educativas de la población. Para el estudio coproparasitológico se recolectó por cada persona, dos muestras de heces obtenidas por evacuación espontánea y en dos días diferentes. El análisis de las heces se realizó mediante un examen directo en solución salina fisiológica y coloración temporal con lugol y por el método de concentración formol-éter

Resultados Se encontró que el $92 \%$ de las personas estaban parasitadas, $92 \%$ de ellas con al menos un patógeno. El poliparasitismo fue muy importante $(89,2 \%)$ encontrándose hasta un máximo de 7 especies por hospedador. La coinfección de protozoarios y helmintos fue frecuente $(64 \%)$. Solo se encontró una frecuencia de teniosis de 0,9\%. Se observó una asociación significativa entre sintomatología y presencia de parásitos $(p<0,05)$ no así, entre síntomas y parásitos potencialmente patógenos a excepción de Trichuris trichura y dolor abdominal. El análisis estadístico no mostró asociación entre las parasitosis y los niveles educativos o hábitos higiénicos sanitarios.

Conclusión La distribución uniforme de la mayoría de las parasitosis intestinales en los cinco grupos de edad evaluados, da cuenta de la exposición a las fuentes de infección en todas las etapas de la vida de los pobladores de Loma Arena.

Palabras Clave: Parasitosis intestinales, prevalencia, factores socioeconómicos, helmintos (fuente: DeCS, BIREME). 


\begin{abstract}
Objectives Determining the prevalence of intestinal parasitism and identifying the associated risk factors in the village of Loma Arena, Bolivar department, Colombia. Methodology The community's sanitary and educational conditions were evaluated by using a questionnaire which was applied to each family group. Two stool samples obtained by spontaneous evacuation, on two different days, were gathered from each participating person for the coproparasitological study. The coprological test involved direct examination in saline physiological solution and temporary staining with Lugol's solution and the formol-ether concentration method.

Results It was found that $92 \%$ of the population was parasitised, $92 \%$ of them with at least one pathogenic parasite. Polyparasitism was very important (89,2 \%); a maximum of 7 species per host was found. Helminth and protozoa coinfection was frequent (64 $\%)$. There was only 0,9\% teniosis prevalence. There was a significant association between symptomatology and parasite presence $(p<0.05)$, though such relationship was not seen with potentially pathogenic parasites (with the exception of Trichuris trichura and abdominal pain). The statistical analysis did not reveal any relationship between parasitism and educational level or sanitary habits.

Conclusion The uniform distribution of most intestinal parasites amongst the five agegroups evaluated showed that people in Loma Arena were evenly exposed to sources of infection in all age-groups.
\end{abstract}

Key Words: Intestinal disease, prevalence, socioeconomic factor, helminth (source: $\mathrm{MeSH}, \mathrm{NLM})$.

L as infecciones parasitarias están distribuidas prácticamente en todo el mundo con alta prevalencia en algunas regiones, afectando a individuos de todas las edades y sexos. Aunque la mortalidad de estas infecciones es relativamente baja, las complicaciones son comunes siendo responsables de al menos el $10 \%$ de las diarreas y en muchos casos requiriendo cuidado hospitalario. En los países afectados, las infecciones parasitarias intestinales están estrechamente relacionadas a los procesos de desarrollo económico y social, ya que la malabsorción, la diarrea y la pérdida de sangre, generan disminución de la capacidad de trabajo y reducción en la velocidad de crecimiento $(1,2)$. En poblaciones urbanas y peri urbanas, la presencia, persistencia y diseminación de los parásitos intestinales (PI) se relacionan en forma directa con las características geográficas y ecológicas específicas del lugar (3), así como con las condiciones de saneamiento básico disponibles y los factores socioeconómicos y culturales $(4,5)$; por lo tanto, su control puede ser un elemento significativo social y político. (2). En Colombia según la última Investigación Nacional de Morbilidad realizada en 1980 por el Instituto Nacional de Salud, se estableció que el 81,8 \% de las personas se encontraban parasitadas y de éstos el $63 \%$ con parásitos patógenos. 
Para la zona de la Costa Atlántica, a esa fecha se reportó una prevalencia de $86,8 \%$, pero se desconoce la frecuencia actual del parasitismo intestinal, a pesar de que la región Atlántica es la que presenta una mayor tasa de mortalidad por diarrea aguda y parasitismo (6,6 por 100000 habitantes) (6). Con el presente estudio se pretendió conocer la prevalencia actual de los diferentes parásitos intestinales en los habitantes de una localidad de la Costa Caribe colombiana

\section{MÉTODOS}

Área de Estudio y Socialización

El estudio se realizó entre febrero y junio de 2004, en el corregimiento de Loma Arena, localidad ubicada en el municipio de Santa Catalina, Departamento de Bolívar, ubicado sobre la carretera al mar a $45 \mathrm{Km}$ de Cartagena, Colombia. El corregimiento se caracteriza por la baja disponibilidad de recursos sanitarios, de salud y económicos. En el marco de la estrategia de la Educación Primaria en Salud, se les informó a la población en general, acerca de las características básicas de la transmisión de las parasitosis y las implicaciones en el ámbito de salud pública que tienen dichas infecciones.

Variables de estudio: Mediante encuesta aplicada por grupo familiar, fueron evaluadas las condiciones socio-sanitarias y educativas de la población con variables como la edad, el género, el nivel educativo, la ocupación, la fuente de agua, la eliminación de excretas y la disposición de basuras. Igualmente a cada persona se le realizó un examen físico general y se le indagó por la presencia de signos y lo síntomas como cefalea, síntomas gastrointestinales, nausea, anorexia, vomito, diarrea, dolor abdominal y prurito anal. Adicionalmente se determinaron las variables independientes presencia o no de parásitos, tipo de parásito y poliparasitismo.

Tipo de muestra y muestreo

Muestra probabilística estratificada. Se realizó un muestreo multietapico. En la primera etapa se seleccionaron de forma aleatoria las manzanas. En la segunda etapa se seleccionaron de forma intencional las casas, en cada manzana siempre se inició de norte a sur y en el sentido contrario a las manecillas del reloj, evaluando una casa de por medio. En la última etapa se seleccionaron los participantes utilizando una tabla de números aleatorios que se aplicó solo en los voluntarios. Con la fórmula de expresión de estimación de una proporción y estimando un sobremuestreo del $10 \%$ se calculó un n de 346 voluntarios. 
Análisis de laboratorio

Para el estudio coproparasitológico se recolectó por cada persona, dos muestras de heces obtenidas por evacuación espontánea y en dos días diferentes. El análisis de las heces se realizó mediante un examen directo en solución salina fisiológica y coloración temporal con Lugol y el método de concentración formol-éter (Ritchie).

\section{Análisis de Datos}

Se hizo análisis descriptivo exploratorio de tipo unidimensional para establecer la distribución de frecuencia asociada con cada variable, se halló la frecuencia absoluta y relativa asociada con cada parámetro estudiado. Se realizaron cruces de variables y se utilizó la prueba chi cuadrado para probar la relación entre éstas. Se ajustó un modelo lineal general con las variables explicativas (parasitosis, edad, sexo, mono y poliparasitismo) para identificar aquellas que se relacionaban significativamente con la variable respuesta. Un nivel de significancia de 0.05 fue utilizado en todas las pruebas. Los datos fueron analizados utilizando el paquete estadístico S.A.S (Stadistical Análysis System) versión 8.2 para Windows.

Consideraciones éticas

A todos los participantes del estudio se les explicó los objetivos del estudio y las implicaciones de los resultados que se obtendrían. Toda persona que aceptó participar en el estudio firmó un consentimiento informado el cual se rige por la resolución 008430 de 1993 del Ministerio de Salud de Colombia. En los menores de edad, el consentimiento informado fue firmado por sus padres o responsables. Se formuló el tratamiento indicado a los casos positivos para parásitos intestinales.

\section{RESULTADOS}

Características sociosanitarias

En el estudio se incluyeron 382 personas, de estas el $60 \%$ en el rango de edad de 15-44 años, el $67 \%$ del sexo femenino, y el $29 \%$ se dedican a los oficios domésticos. La principal fuente de agua es el carro repartidor (80 \%) y la mayoría de las personas (78 \%) consume el agua sin tratar. El 45\% elimina las excretas a campo abierto. El $77 \%$ de las personas quema la basura. El $86 \%$ convive con animales, principalmente con perros (18\%). Adicionalmente se encontró que el 12 \% de la población es analfabeta y 39 \% cursó primaria incompleta.

Análisis parasitológico

De las 382 personas estudiadas, 352 (92 \%) presentaron una o varias especies 
de parásitos intestinales, y de estas el 92 \% presentaba al menos un parasito potencialmente patógeno. El 89,2 \% presentó más de una especie parasitaria (poliparasitismo) con un máximo de 7 especies por hospedador. Se identificaron 8 especies de protozoarios intestinales incluyendo patógenos y comensales y 7 especies de helmintos. Las mayores frecuencias de E. coli y el complejo $E$ histolityca/E. dispar, entre los protozoarios y Ascaris lumbricoides y Trichuris trichiura entre los los helmintos, se observan en la Tabla 1.

Tabla 1. Prevalencia de parásitos intestinales en población general del corregimiento de Loma Arena Santa Catalina. 2004

\begin{tabular}{lcc}
\hline Especie parasitaria & $\mathrm{N}^{\circ}$ & $\%$ \\
\hline Entamoeba coli & 210 & 60 \\
Entamoeba histolityca/dispar & 191 & 54 \\
Endolimax nana & 125 & 36 \\
Blastocystis hominis & 103 & 29 \\
Iodamoeba butschlii & 72 & 21 \\
Giardia duodenalis & 61 & 17 \\
Trichomona hominis & 3 & 0,9 \\
Ciclospora sp & 2 & 0,6 \\
Ascaris lumbricoides & 196 & 56 \\
Trichuris trichiura & 185 & 53 \\
Uncinaria & 21 & 6 \\
Hymenolepis nana & 14 & 4 \\
\hline Strongyloides stercolaris & 11 & 3 \\
Taenia sp & 3 & 0,9 \\
Enterobius vermicularis & 2 & 0,6 \\
\hline
\end{tabular}

Prevalencia de parásitos según sexo

Se observó que el $93 \%$ de las mujeres y el $90 \%$ de los hombres estaban parasitados y de estos el 91\% de las mujeres y el 94\% de los hombres con al menos un parásito potencialmente patógeno. La prueba chi-cuadrado mostró asociación $(p<0,05)$ entre las variables en estudio (sexo y parasitosis). El protozoario más frecuente en las mujeres fue Entamoeba coli y en los hombres Entamoeba histolytica/dispar y el helminto más frecuente en ambos sexos fue Ascaris lumbricoides. Sólo se obtuvo diferencia estadísticamente significativa, $(\mathrm{p}<0,05)$ en la prevalencia de E. coli y uncinaria las cuales fueron mayor en el sexo femenino y en el masculino respectivamente.

Prevalencia de parásitos según edad

Se encontró una prevalencia del $90 \%$ en el rango de edad de 0-5 años, $98 \%$ en el de 5-14 años, $93 \%$ en el de 15-44, $87 \%$ en el de 45-60 años y $89 \%$ en los mayores de 60, sin hallarse una diferencia significativa ( $>>0,05)$. En los grupos de 0-5 años y de 6-14 se encontró que el $100 \%$ de las personas parasitadas presentaban al menos un parásito potencialmente patógeno. No se observaron diferencias estadísticamente significativas $(p>0,05)$ entre los grupos de edad a 
excepción de Trichuris trichiura que tuvo mayor prevalencia entre los grupos de 0-4 y 5-14 años, en comparación con el grupo etario de 45-60 años, siendo esta diferencia, estadísticamente significativa $(\mathrm{p}<0,05)$.

Prevalencia de parásitos por sexo y edad

Se encontró una prevalencia muy similar entre las mujeres y los hombres en todos los grupos de edad $(\mathrm{p}>0,05)$. Trichuris trichiura fue la especie más frecuente en ambos sexos del rango de los 5 a 14 años (Tabla 2).

Tabla 2. Distribución de las parasitosis por rangos de edad y sexo en población general del corregimiento de Loma Arena Santa Catalina. 2004

\begin{tabular}{|c|c|c|c|c|c|c|c|c|c|c|}
\hline \multirow{3}{*}{ Especie parasitaria } & \multicolumn{5}{|c|}{ Mujeres \% } & \multicolumn{5}{|c|}{ Hombres \% } \\
\hline & \multicolumn{10}{|c|}{ Grupo etario } \\
\hline & 1 & 2 & 3 & 4 & 5 & 1 & 2 & 3 & 4 & 5 \\
\hline Entamoeba col & 67 & 47 & 60 & 59 & 47 & 46 & 48 & 51 & 14 & 46 \\
\hline Entamoeba histolitycerdispar & 33 & 47 & 49 & 54 & 53 & 46 & 52 & 51 & 14 & 54 \\
\hline Endollmax nane & 22 & 29 & 34 & 28 & 20 & 23 & 48 & 31 & 21 & 38 \\
\hline Blastocystis hominis & 11 & 15 & 32 & 31 & 33 & 8 & 24 & 25 & 14 & 15 \\
\hline fodamoeba butschW & 11 & 15 & 16 & 31 & 13 & a & 38 & 21 & 0 & 15 \\
\hline Giardia duodenalls & 0 & 6 & 14 & 18 & 7 & 31 & 24 & 25 & 7 & 0 \\
\hline Trichamona haminis & 0 & 0 & 0 & 0 & 7 & 0 & 0 & 1 & 0 & 0 \\
\hline Ciclospora sp & 0 & 0 & 1 & 0 & 0 & 0 & 0 & 1 & 0 & 0 \\
\hline Asceris fumbricaiches & 44 & 59 & 50 & 54 & 60 & 77 & 52 & 46 & 14 & $3 \mathrm{~B}$ \\
\hline Trichuris trichiera & 56 & 76 & 45 & 33 & 60 & 77 & 81 & 37 & 14 & 23 \\
\hline Uncinaria & 0 & 0 & 5 & 0 & 0 & 8 & 5 & 12 & 14 & 8 \\
\hline Hymenolepis nana & 0 & 9 & 4 & 0 & 0 & 15 & 5 & 1 & 0 & 0 \\
\hline Strongyiaides stercolanis & 0 & 3 & 4 & 0 & 0 & 0 & 5 & 3 & 0 & 0 \\
\hline Taenva sp & D & 3 & 1 & D & 0 & 0 & 0 & 1 & 0 & 0 \\
\hline Enterabius vermicularis & D & 0 & 1 & 3 & 0 & 0 & o & 0 & D & 0 \\
\hline
\end{tabular}

Grupo etario: 1: 0-4 años, 2: 5-14 años, 3:15-44 años, 4: 45-60 años, 5: >60 años

Asociación entre la presencia de síntomas y parásitos gastrointestinales

De la población general, el 43 \% manifestaron presentar algún síntoma gastrointestinal, como dolor abdominal (34\%), diarrea (18\%), anorexia (12\%) y nauseas (6,3 \%). De los sintomáticos, el $91 \%$ estaban parasitadas y al $87 \%$ se les observó al menos un parásito potencialmente patógeno. De otro lado entre las personas que no manifestaron tener síntomas, el 89 \% estaban parasitadas y de éstas el $83 \%$ con al menos un parásito potencialmente patógeno. Se observó una asociación significativa entre sintomatología y presencia de parásitos $(p<0,05)$ no así, entre síntomas y parásitos potencialmente patógenos.

\section{DISCUSIÓN}

Si bien la prevalencia de las principales parasitosis intestinales del mundo no ha cambiado, ellas han aumentado en términos absolutos debido al crecimiento de la población. 
En este estudio realizado a una muestra significativa de la población de Loma Arena, se encontró que el 92 \% de las personas estaban parasitadas y de éstas al 92 \% se les observó al menos un patógeno potencial. El porcentaje de personas con poliparasitismo fue muy importante $(89,2 \%)$ y la coinfección de protozoarios y helmintos fue frecuente (64\%), situaciones que siempre se han relacionado con las condiciones ambientales y el comportamiento humano. Sin embargo en este caso, el análisis estadístico no mostró asociación entre las parasitosis y los niveles educativos o hábitos higiénicos sanitarios, probablemente debido a la homogeneidad en los bajos grados educativos de la comunidad y en las malas condiciones higiénicas.

Por otra parte, la distribución uniforme de la mayoría de las parasitosis intestinales en los cinco grupos de edad evaluados, da cuenta de la exposición a las fuentes de infección en todas las etapas de la vida de los pobladores de Loma Arena, sin encontrarse diferencias significativas entre hombres y mujeres, lo cual sugiere la existencia de conductas similares entre los géneros, que posibilitan la prevalencia equitativa de las parasitosis

La alta prevalencia de helmintos encontrada en este estudio concuerda con lo reportado en un estudio de la costa pacífica (7), no así con publicaciones de otras regiones del país $(8,9)$, estas diferencias podrían estar relacionadas con las particulares condiciones climáticas y las características del suelo de las regiones costeras estudiadas, ya que la temperatura, humedad y/o vientos influyen en la distribución de los geohelmintos (10). Llaman la atención los casos de teniosis, dos de los cuales se presentaron en el grupo etario de 5-14 años de edad, sobre todo sí se tiene en cuenta que la infección con este helminto es el principal factor de riesgo para la aparición de neurocisticercosis.

Los resultados de prevalencia de protozoarios de este estudio difieren de los publicados por otros autores en varias regiones colombianas $(7-9,11)$ especialmente Giardia lamblia que no fue relevante aún en población infantil. Aunque en general se encontró una importante prevalencia de parásitos comensales, la mayor frecuencia correspondió a E. coli (60 \%) y E. nana (36 \%), lo cual guarda relación con lo expuesto en otras investigaciones. $(8,11,12)$. Esta prevalencia de protozoarios comensales carece de importancia clínica pero tiene importancia epidemiológica, pues es referente de la contaminación con materia fecal de los alimentos y del agua de consumo, siendo éstos, los mismos vehículos para la transmisión de los otros protozoarios potencialmente patógenos también encontrados en esta población, como es el caso de Entamoeba histolityca/ dispar (54\%) 
El primer lugar de las helmintiasis lo ocupó A. lumbricoides con una prevalencia de $56 \%$, seguido de $T$. trichiura con una prevalencia de $53 \%$, esto es de particular importancia considerando que en algunos estudios se ha observado una correlación significativa entre el grado de infección con geolhelmintos $\mathrm{y}$ algunas funciones cognitivas que pueden ser al menos parcialmente reversibles luego de un tratamiento con antihelmínticos. A. lumbricoides, afecta principalmente los niveles de habilidad verbal; así como el desempeño en la producción de respuestas ante un estimulo (prueba de Stroop), mientras que T. trichiura se ha asociado con la disminución de la capacidad de la atención y de la memoria a corto y largo plazo (13-15). Las mencionadas funciones cognitivas son indicadores fundamentales en la evaluación del desarrollo de lactantes, preescolares y escolares, población que en este estudio presentó predominantemente grados moderado e intenso de infección. La relevancia de las helmintiasis en la población lactante ya había sido reportada en otros estudios colombianos $(7,16)$.

Adicionalmente T. trichiura tiene gran importancia clínica ya que la infección extrema con esta especie parasitaria puede producir pérdida hemática de origen intestinal con la consiguiente anemia (17), valores bajos de peso para la talla (7), apendicitis o en niños y mujeres parturientas prolapso rectal.

En algunos parásitos intestinales el potencial patogénico está bien establecido, mientras que no es así en otros. El curso clínico de la infección con parásitos puede variar ampliamente desde asintomático hasta complicaciones secundarias graves, dependiendo del número, tipo e intensidad de las infecciones, así como del estado inmunitario de la persona infectada (18). Con respecto a la sintomatología gastrointestinal en la población estudiada, se puede señalar que aunque se halló una asociación estadística entre los síntomas y el hallazgo de algún parásito, patógeno o no, también se encontró de manera significativa, población parasitada asintomática, de ahí que es importante tener en cuenta dos conductas a seguir en esta zona geográfica, ya sea realizar el estudio coproparasitológico a los individuos que manifiesten tener alguno de los síntomas mencionados e incluso en población asintomática, pero con factores epidemiológicos determinantes.

La asociación entre síntomas específicos y especies parasitarias ha sido inconstante en las diversas descripciones: mientras que algunos autores no han encontrado ninguna asociación entre síntomas e infección parasitaria intestinal (19) o han concluido que la presencia de síntomas tiene un bajo valor predictivo positivo para la presencia de parásitos patógenos (20), otros como 
Utzinger J. et al, han reportado la diarrea como un síntoma útil para la identificación de infecciones por E. hystolitica/dispar, (21) y Miller et al han asociado el dolor abdominal con la presencia de Áscaris lumbricoides y de Blastocystis hominis (22), este último hallazgo, coincide con las apreciaciones de El-Shazly et al (23). En el presente estudio no se encontró ningún síntoma específicamente asociado a infección con B. hominis, lo cual no apoya su consideración como un importante patógeno emergente a nivel mundial (22). $\mathrm{Al}$ igual que en otros estudios (22), no se encontró a E. histolytica/dispar asociaciada con sintomatología gastrointestinal. En cuanto al resto de parásitos reconocidos como patógenos y a diferencia de otros estudios, se encontró asociación estadísticamente significativa entre la infección con T. trichiura y la presencia de dolor abdominal (21-23).

Los hallazgos de este estudio responden a un complejo círculo vicioso, que implica el detrimento de la salud de los individuos, disminuyendo sus habilidades productivas y limitando sus posibilidades de ingreso a niveles de educación que moldeen sus hábitos higiénicos. La importancia de las parasitosis intestinales reside tanto en el efecto directo que ejercen sobre la salud del individuo, como en el hecho de que éstas constituyen índices de condiciones sanitarias precarias en la comunidad, por lo tanto es necesario establecer la prevalencia específica de las PI, en determinadas zonas geográficas para visualizar un panorama localizado de la situación y así entablar las correspondientes acciones de salud pública y de atención médica dirigidas a los puntos críticos identificados *

Agradecimientos. Al Comité de Investigaciones (CODI) de la Universidad de Antioquia, a la Comunidad de Loma Arena, al Centro de Investigaciones de la Universidad Metropolitana de Barranquilla, a la Alcaldía del municipio de Santa Catalina y a Concesión Salinas de Galerazamba.

\section{REFERENCIAS}

1. McDonald V. Parasites in the gastrointestinal tract. Parasite Immunol 2003;25:231-4.

2. WHO. Prevention and control of intestinal parasitic infections. Tech Re Ser: WHO Expert Committee; 1987. Report No. 749.

3. Soriano S, ManacordaA, Pierangeli N, Navarro M. Intestinal parasitosis in relation to socioeconomic factors and habitat conditions in children of Neuquén, Patagonia, Argentina. Parasitol Latinoam 2005;60(3-4):154-161.

4. Al-Shammari S, Khoja T, El-Khwasky F, Gad A. Intestinal parasitic diseases in Riyadh, Saudi Arabia: prevalence, sociodemographic and environmental associates. Trop Med Int Health 2001;6(3):184-9. 
5. Gamboa MI, Basualdo JA, Cordoba MA, Pezzani BC, Minvielle MC, Lahitte HB. Distribution of intestinal parasitoses in relation to environmental and sociocultural parameters in La Plata, Argentina. J Helminthol 2003;77(1):15-20.

6. Orozco AJM. Gestión del DADIS 2005-2006 [Internet]. Resultados en Salud Pública. En: Evaluación de la aplicación de políticas públicas de salud en la ciudad de Cartagena. Eumet.net. 2006. Disponible en http://www.eumed.net/libros/2006c/199/index.htm. Consultado: Agosto 2007

7. Alvarado BE, Vasquez LR. Social determinants, feeding practices and nutritional consequences of intestinal parasitism in young children. Biomedica 2006;26(1):82-94.

8. Giraldo-Gomez JM, Lora F, Henao LH, Mejia S, Gomez-Marin JE. Prevalence of giardiasis and intestinal parasites in pre-school children from homes being attended as part of a state programme in Armenia, Colombia. Rev Salud Pública (Bogota) 2005;7(3):327-38.

9. Cortés JR, Salamanca L, Sanchez M, Vanegas F, Sierra PA. Parasitismo y estado nutricional en niños preescolares de intituciones de Santa Fé de Bogotá. Revista Colombiana de Pediatria 34(4); 1999.

10.Mabaso ML, Appleton CC, Hughes JC, Gouws E. The effect of soil type and climate on hookworm (Necator americanus) distribution in KwaZulu-Natal, South Africa. Trop Med Int Health 2003;8(8):722-7.

11. Ordonez LE, Angulo ES. Malnutrition and its association with intestinal parasitism among children from a village in the Colombian Amazonian region. Biomedica 2002;22:486-98.

12. Sanzón F, Vela JC, Valencia HF, Montenegro L. Una Estrategia antiparasitaria original en Arboleda, Nariño. Colombia Médica 1999;30(3):112-117.

13. Levav M, Mirsky AF, Schantz PM, Castro S, Cruz ME. Parasitic infection in malnourished school children: effects on behaviour and EEG. Parasitology 1995;110 ( Pt 1):103-11.

14. Nokes C, Grantham-McGregor SM, Sawyer AW, Cooper ES, Robinson BA, Bundy DA. Moderate to heavy infections of Trichuris trichiura affect cognitive function in Jamaican school children. Parasitology 1992;104 ( Pt 3):539-47.

15. Ezeamama AE, Friedman JF, Acosta LP, Bellinger DC, Langdon GC, Manalo DL, et al. Helminth infection and cognitive impairment among Filipino children. Am J Trop Med Hyg 2005;72(5):540-8.

16. Fernandez-Nino JA, Reyes-Harker P, Moncada-Alvarez LI, Lopez MC, Chaves M, Knudson A, et al. Soil-transmitted helminth trends and prevalence in La Virgen, Colombia 1995-2005. Rev Salud Pública (Bogotá) 2007;9(2):289-96.

17. Botero JHC, Montoya MN, Hurtado MI, Ocampo NE, Agudelo GM, Cardona OL, Posada M, Marín C, Escobar LM, Cuéllar F, Díaz A, Muñoz AL, Berrío M. Correa MC, López CP. Anemia por deficiencias de hierro y su asociación con los parásitos intestinales, en escolares y adolescentes matriculados en instituciones oficiales y privadas de Medellín, 1997-1998. Acta Médica Colombiana 2002;27(1):7-14.

18. Higgins DA, Jenkins DJ, Kurniawan L, Purnomo, Harun S, Juwono SS. Human intestinal parasitism in three areas of Indonesia: a survey. Ann Trop Med Parasitol 1984;78(6):637-48.

19. Limoncu ME, Kurt O, Gumus M, Kayran E, Balcioglu IC, Dinc G, et al. Is there an association between clinical symptoms and intestinal parasitic infections? Int J Clin Pharmacol Res 2005;25(3):151-4.

20. Buchwald D, Lam M, Hooton TM. Prevalence of intestinal parasites and association with symptoms in Southeast Asian refugees. J Clin Pharm Ther 1995;20(5):271-5.

21. Utzinger J, N'Goran EK, Marti HP, Tanner M, Lengeler C. Intestinal amoebiasis, giardiasis and geohelminthiases: their association with other intestinal parasites and reported intestinal symptoms. Trans R Soc Trop Med Hyg 1999;93(2):137-41.

22. Miller SA, Rosario CL, Rojas E, Scorza JV. Intestinal parasitic infection and associated symptoms in children attending day care centres in Trujillo, Venezuela. Trop Med Int Health 2003;8(4):342-7.

23. El-Shazly AM, Abdel-MagiedAA, El-Beshbishi SN, El-Nahas HA, Fouad MA, Monib MS. Blastocystis hominis among symptomatic and asymptomatic individuals in Talkha Center, Dakahlia Governorate, Egypt. J Egypt Soc Parasitol 2005;35(2):653-66. 